\title{
Electric-Field-Induced Reversible Phase Transitions in a Spontaneously lon-Intercalated 2D Metal Oxide
}

\author{
Hamid Reza Rasouli, Jeongho Kim, Naveed Mehmood, Ali Sheraz, Min-kyung Jo, Seungwoo Song, \\ Kibum Kang,* and Talip Serkan Kasirga*
}

Cite This: Nano Lett. 2021, 21, 3997-4005

Read Online

\section{ACCESS | Lill Metrics \& More | 回 Article Recommendations ｜（） Supporting Information}

ABSTRACT: Electric field driven reversible phase transitions in two-dimensional (2D) materials are appealing for their potential in switching applications. Here, we introduce potassium intercalated $\mathrm{MnO}_{2}$ as an exemplary case. We demonstrate the synthesis of largearea single-crystal layered $\mathrm{MnO}_{2}$ via chemical vapor deposition as thin as $5 \mathrm{~nm}$. These crystals are spontaneously intercalated by potassium ions during the synthesis. We showed that the charge transport in $2 \mathrm{D} \mathrm{K}-\mathrm{MnO}_{2}$ is dominated by motion of hydrated potassium ions in the interlayer space. Under a few volts bias, separation of potassium and the structural water leads to formation of different phases at the opposite terminals, and at larger biases $\mathrm{K}$ $\mathrm{MnO}_{2}$ crystals exhibit reversible layered-to-spinel phase transition. These phase transitions are accompanied by electrical and optical

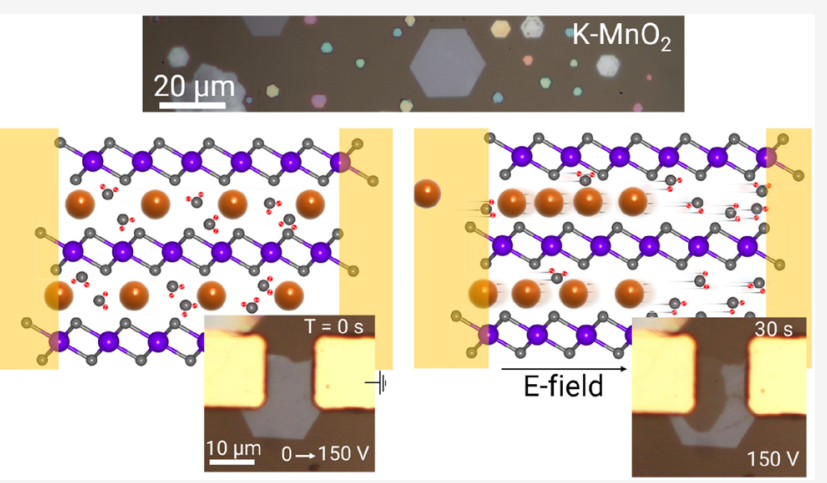
changes in the material. We used the electric field driven ionic motion in $\mathrm{K}-\mathrm{MnO}_{2}$ based devices to demonstrate the memristive capabilities of two terminal devices.

KEYWORDS: $2 d$ materials, phase transitions, ion transport, $\mathrm{MnO}_{2}$, real time optical chemical vapor deposition

$\mathrm{E}$ lectric field control of phase transitions is long sought for switching applications in electronics and optoelectronics. Recently, structural phase transitions accompanied by electronic changes in two-dimensional (2D) materials have been shown to be suitable candidates in voltage controlled applications. ${ }^{1,2}$ One way to achieve electric field control in electrical properties of a material is by moving ions across the interlayer space to induce structural distortions. ${ }^{3,4}$ Such ion intercalation engineering of $2 \mathrm{D}$ materials ${ }^{5,6}$ may be a key to enabling novel technologies in energy storage, ${ }^{7,8}$ electronics, ${ }^{9,10}$ optoelectronics, ${ }^{11-15}$ neuromorphic computing, ${ }^{4}$ and magnetic information storage and processing. ${ }^{16}$ However, challenges such as wrinkling and distortion of the crystal in extrinsic ion intercalation through the edges of the $2 \mathrm{D}$ materials limit its applicability even for proof-of-concept demonstrations. One way around this challenge would be using spontaneously ion intercalated materials during the synthesis. Manganese dioxide $\left(\mathrm{MnO}_{2}\right)$ can be an alternative as it can host a variety of cations and exists in layered form. However, no large area 2D crystals of $\mathrm{MnO}_{2}$ has been demonstrated so far.

$\mathrm{MnO}_{2}$ has been studied for various applications in catalysis, ${ }^{17,18}$ supercapacitor and battery applications, ${ }^{19,20}$ water extraction and splitting, ${ }^{21}$ and others ${ }^{22}$ as it is a lowcost, environmentally friendly alternative. $\mathrm{MnO}_{2}$ exhibits diverse polymorphism when synthesized via hydrothermal methods. ${ }^{23-26}$ Depending on the specific framework formed by the edge- and corner-sharing metal-oxide octahedra, $\left[\mathrm{MnO}_{6}\right]$, the polymorphs can be classified as layered (2D) or tunnel (1D) $\mathrm{MnO}_{2}$. Both $1 \mathrm{D}$ and $2 \mathrm{D}$ structures can host cations such as $\mathrm{Li}^{+}, \mathrm{Na}^{+}, \mathrm{K}^{+}$, and so forth that stabilize the structures along with the structural water. ${ }^{26,27}$ Phase transition from $2 \mathrm{D}$ to $1 \mathrm{D}$ structures is possible via motion of $\mathrm{Mn}$ atoms from intralayer space to the interlayer positions. ${ }^{28}$ Besides the transitions among layered and tunnel polymorphs, it is also possible to induce phase transitions across $\mathrm{MnO}_{2}$ and $\mathrm{Mn}_{3} \mathrm{O}_{4}$ electrochemically ${ }^{19,29-32}$ and this phase transition is relevant to the applications pertaining to $\mathrm{MnO}_{2}$. Representative crystal structure schematics of the $\mathrm{MnO}_{2}$ polymorph classes and spinel- $\mathrm{Mn}_{3} \mathrm{O}_{4}$ are depicted in Figure 1a. Previous studies demonstrate that $\mathrm{K}-\mathrm{MnO}_{2}$ layers obtained via hydrothermal synthesis can be delaminated atomically thin flakes. ${ }^{33-35}$ However, the crystal sizes obtained via liquid or mechanical exfoliation methods are far too small for practical single crystal device applications and they exhibit poor crystalline quality.

Received: February 23, 2021

Revised: April 16, 2021

Published: April 21, 2021 


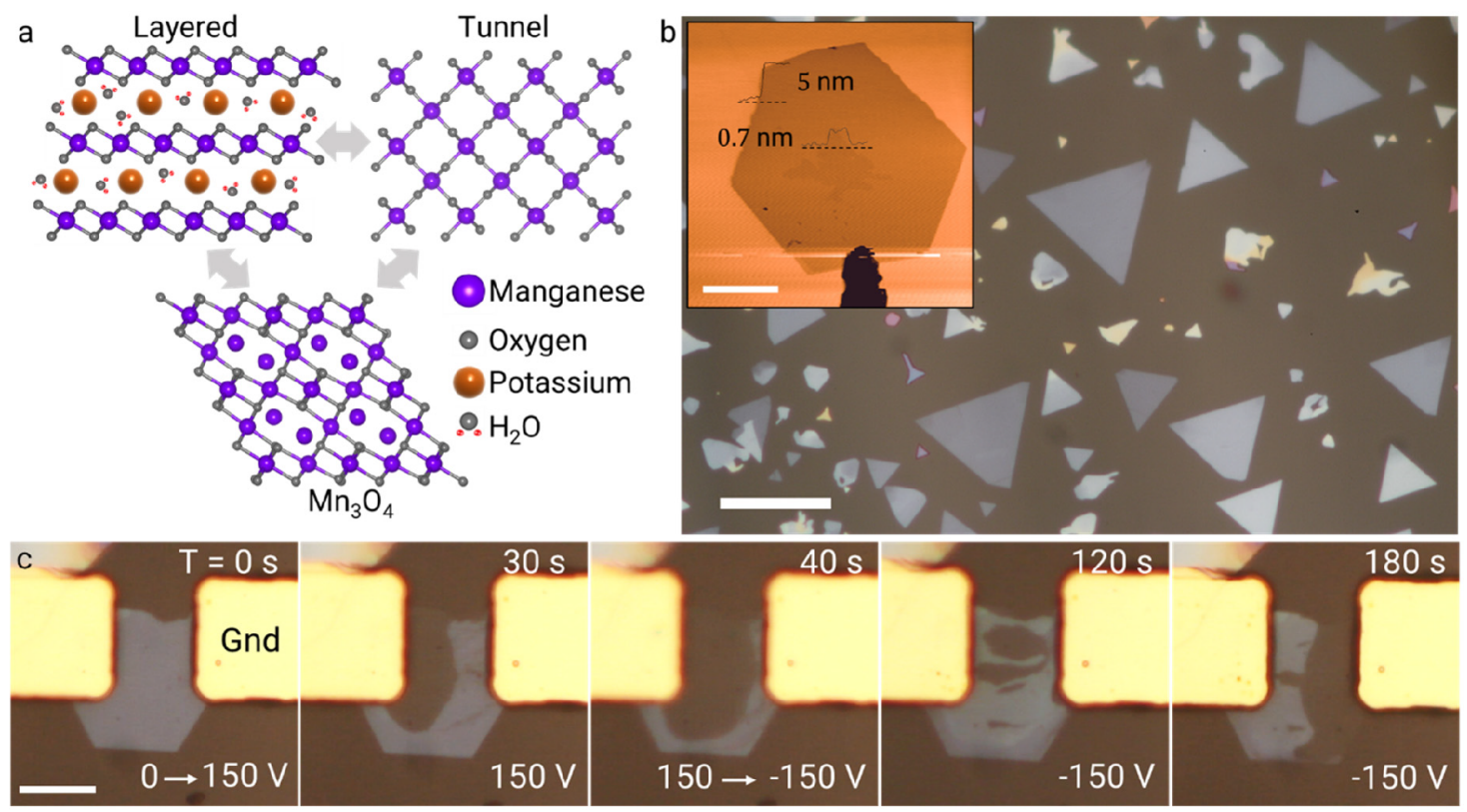

Figure 1. (a) Representative schematics of layered and tunnel polymorphs of $\mathrm{MnO}_{2}$ and, $\mathrm{Mn}_{3} \mathrm{O}_{4}$. Phase transition among various polymorphs is possible. $\mathrm{Mn}^{2+}$ ions in $\mathrm{Mn}_{3} \mathrm{O}_{4}$ schematic are depicted without bonds for clarity. (b) Optical microscope image of $2 \mathrm{D} \mathrm{K}-\mathrm{MnO}_{2}$ crystals on sapphire substrate with the inset showing an AFM map of a $5 \mathrm{~nm}$ thick $2 \mathrm{D} \mathrm{K}-\mathrm{MnO}_{2}$ crystal. An overgrowth at the center of the crystal shows the monolayer thickness of $0.7 \mathrm{~nm}$. Scale bar for the inset is $5 \mu \mathrm{m}$ and for the main image $20 \mu \mathrm{m}$. Note the preferred growth orientation of the crystal, indicating the epitaxial relation with the sapphire substrate. (c) Series of optical microscope images taken during biasing of a two-terminal device of $\mathrm{K}-\mathrm{MnO}_{2}$. The first image shows the moment just before applying $150 \mathrm{~V}(T=0 \mathrm{~s})$ and the consecutive images show the evolution of optical contrast of the crystal. After $30 \mathrm{~s}$, more than half of the crystal shows less contrast with respect to the substrate. At $40 \mathrm{~s}$, the bias is reversed, $-150 \mathrm{~V}$ with respect to ground is applied. Within $80 \mathrm{~s}$ a phase with different contrast than the pristine crystal appears. Finally, the less contrasty phase appears near the ground electrode.

Here, we introduce $\mathrm{K}-\mathrm{MnO}_{2}$ as a spontaneously ion intercalated $2 \mathrm{D}$ material that can be synthesized as large area atomically thin crystals for the first time. We synthesized single crystals of lateral size up to $100 \mu \mathrm{m}$ on a c-cut sapphire substrate using real-time observation chemical vapor deposition method (RTO-CVD). ${ }^{36}$ Figure $1 \mathrm{~b}$ shows typical crystals on sapphire substrate. Atomic force microscopy (AFM) height trace maps reveal $\sim 0.7 \mathrm{~nm}$ thick atomic layers of $2 \mathrm{D} \mathrm{K}-\mathrm{MnO}_{2}$ as shown in the inset of Figure $1 \mathrm{~b}$. We show that $2 \mathrm{D} \mathrm{K}-\mathrm{MnO}_{2}$ is an ionic conductor with in-plane motion of ions. Potassium ion motion along with the water absorbed from the ambient contributes significantly to the charge transport. The ion migration and water intercalation/deintercalation from the ambient in $\mathrm{K}-\mathrm{MnO}_{2}$ results in reversible phase transitions of the crystal structure among layered and spinel phases and causes variations in the ionic content, accompanied by an optical contrast change (Figure 1c, SI Video 1). By utilizing the ion migration and the phase transitions, we demonstrate that $\mathrm{K}-\mathrm{MnO}_{2}$ can be used as an active medium in neuromorphic device applications and explained the mechanisms leading to the memristive properties of $\mathrm{K}-\mathrm{MnO}_{2}$ devices.

The synthesis of $\mathrm{K}-\mathrm{MnO}_{2}$ is based on melting of the metal oxide precursor mixed with the potassium salt on c-cut sapphire substrate for an epitaxial growth. $\mathrm{KI}$ and $\mathrm{MnO}_{2}$ powders are milled together in a fine powder in 5:1 ratio and placed on the growth substrate. The growth takes place under ambient atmosphere in a sealed chamber at $640{ }^{\circ} \mathrm{C}$. Once the crystals of desired size are observed optically, the substrate heater is turned off and the substrates are naturally cooled down to the room temperature within a few minutes. SI Video 2 shows the real-time crystal growth. Details of the growth are given in the Supporting Information (SI). The same recipe is transferred to conventional CVD and similar high-quality crystals are obtained (Figure S1). As synthesized $\mathrm{K}-\mathrm{MnO}_{2}$ crystals are exceptionally stable under the ambient conditions as studied by Raman and electrical measurements over four month-old samples (Figure S2).

The crystallinity and the crystal structure of $2 \mathrm{D}$ nanosheets are studied by X-ray diffraction (XRD). The sharp diffraction peaks in $\theta-2 \theta$ scan (Figure $2 \mathrm{a}$ ) are located at $12.9^{\circ}$ and $25.6^{\circ}$ corresponding to (001) and (002) planes of $\mathrm{K}-\mathrm{MnO}_{2}$, respectively. These $\mathrm{XRD}$ scans agree with the Birnessite structure, namely the $\delta$ - $\mathrm{MnO}_{2}$ (JCPDS card No. 801098). ${ }^{37-39}$ The interlayer spacing calculated from the (001) plane is $\sim 0.69 \mathrm{~nm}$. This is consistent with the thickness of an overgrowth layer obtained via AFM. Absence of other diffraction peaks is due to the parallel orientation of the crystal surfaces with respect to the c-cut sapphire surface (0006). Monoclinic and hexagonal layered crystal structures of $\delta$ - $\mathrm{MnO}_{2}$ has been reported earlier. ${ }^{40}$ Due to the absence of other characteristic peaks, it is not possible to determine whether the layered structure belongs to the monoclinic or the hexagonal crystal system solely based on the XRD. Thus, selected-area electron diffraction (SAED) patterns are obtained using transmission electron microscope (TEM) and confirmed that the $2 \mathrm{D} \mathrm{K}-\mathrm{MnO}_{2}$ matches with the monoclinic crystal system (Figure 2b). ${ }^{41}$ Supercell reflections in our SAED patterns are consistent with the ordered distribution of the interlayer species.

Two-dimensional $\mathrm{K}-\mathrm{MnO}_{2}$ crystal phase is also confirmed by the Raman spectroscopy (Figure 2c). There are nine Raman modes at 196, 236, 280, 406, 470, 506, 557, 577, and $636 \mathrm{~cm}^{-1}$ that can be attributed to the monoclinic $\mathrm{K}-\mathrm{MnO}_{2}$ (ref 42). The $557 \mathrm{~cm}^{-1}$ attributed to the out-of-plane vibrational modes of 

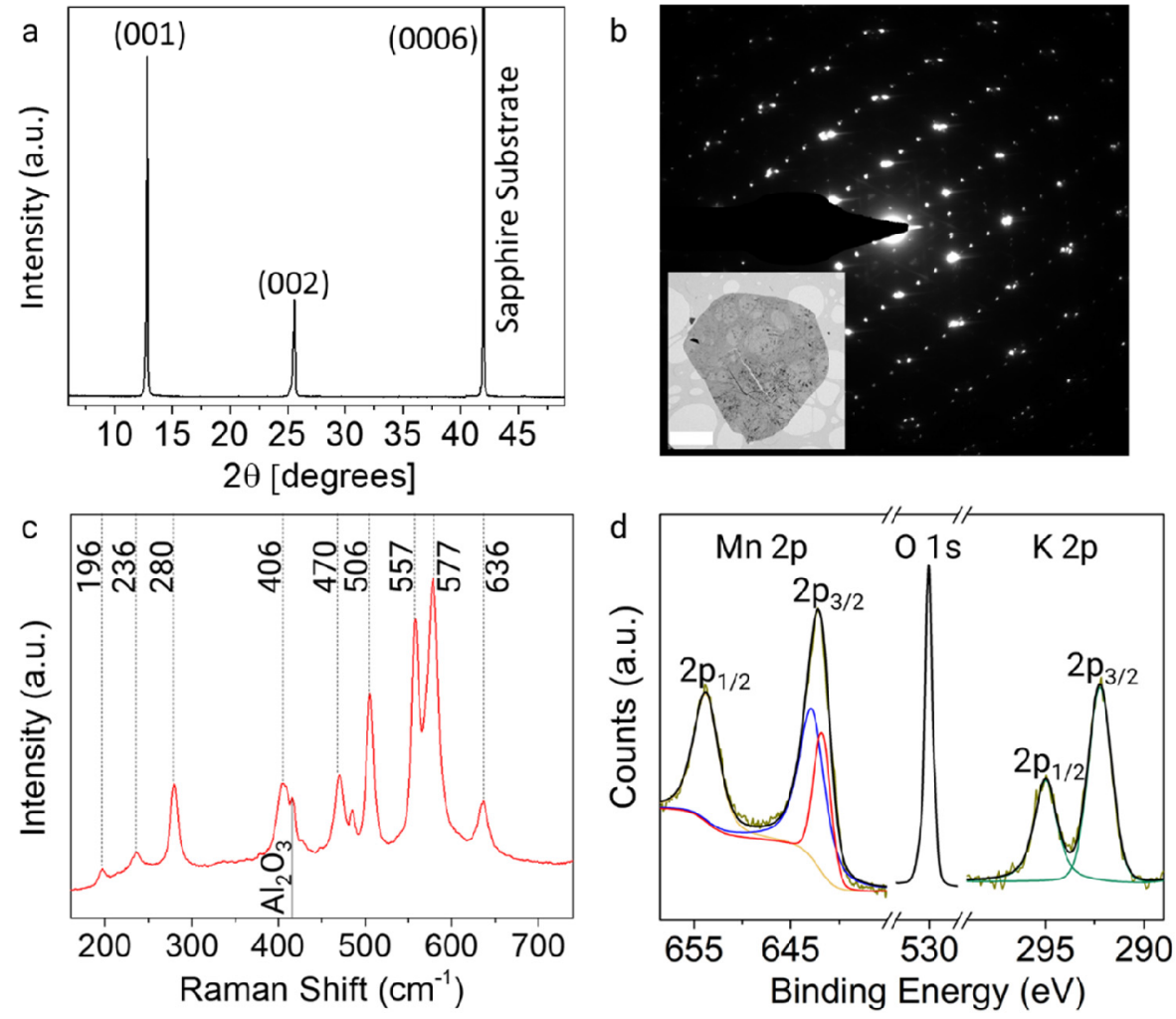

Figure 2. (a) XRD pattern from as synthesized crystals on sapphire shows narrow peaks corresponding to the planes parallel to the substrate surface. Consistent with the AFM, interlayer spacing calculated from the $2 \theta$ angle of (001) is $\sim 0.69 \mathrm{~nm}$. (b) SAED pattern agrees with the monoclinic crystal structure. Supercell reflections attributed to the interlayer cations are also evident. Inset shows a wide-field TEM image of a 2D $\mathrm{K}-\mathrm{MnO}_{2}$ over a holey carbon grid. Scale bar in the inset is $4 \mu \mathrm{m}$. (c) Raman spectrum of a $2 \mathrm{D} \mathrm{K}-\mathrm{MnO}_{2}$ crystals on sapphire substrate shows nine Raman modes marked with dashed lines. Raman peak from the substrate is marked with a solid line. (d) XPS surveys for Mn 2p, O 1s, and K 2p peaks. Mn $2 p$ survey shows that the $\mathrm{Mn}$ is in +3 and +4 oxidation states as determined by two Gaussian functions that can be fitted to $2 p_{3 / 2}$ peak.
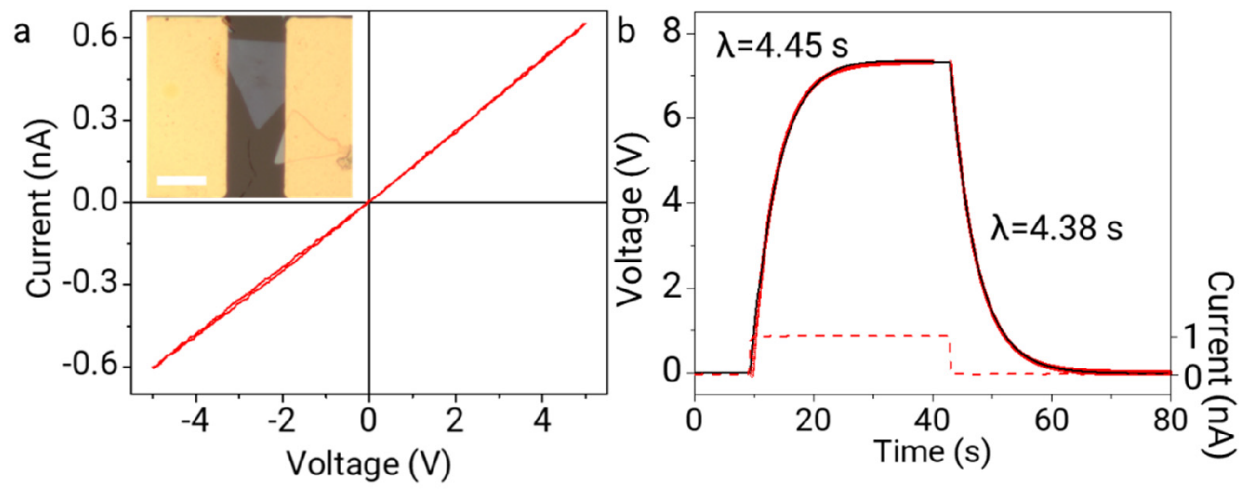

C

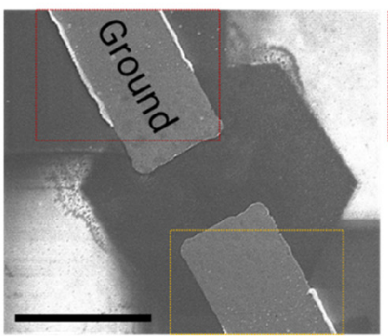

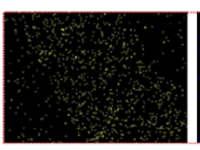

$\mathrm{K}$

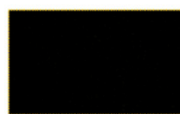

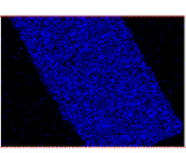

Au

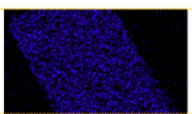

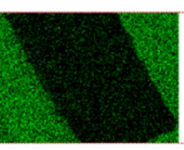

Al

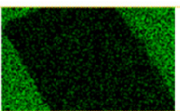

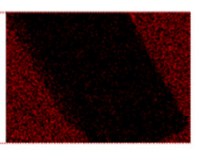

0

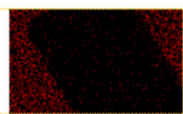

Figure 3. (a) $I-V$ cycle of a device shown in the inset. Scale bar for the inset is $10 \mu \mathrm{m}$. (b) $V-t$ scan under constant current shows an exponentially decaying increase and decrease in the voltage response across the electrodes (shown as solid black line). The exponential fit to the rising and decaying parts are given with open red circles. $\lambda$ is the time constant in the exponential function used for fitting the experimental data. Dashed line shows the current applied at various time. (c) SEM micrograph and the EDX maps corresponding to regions in dashed rectangles at the upper and lower contacts of a positively biased device are given. SEM image suffered from heavy charging due to the sapphire substrate. Ground contact shows the presence of $\mathrm{K}$ while the other contact lacks. Scale bar is $20 \mu \mathrm{m}$. 

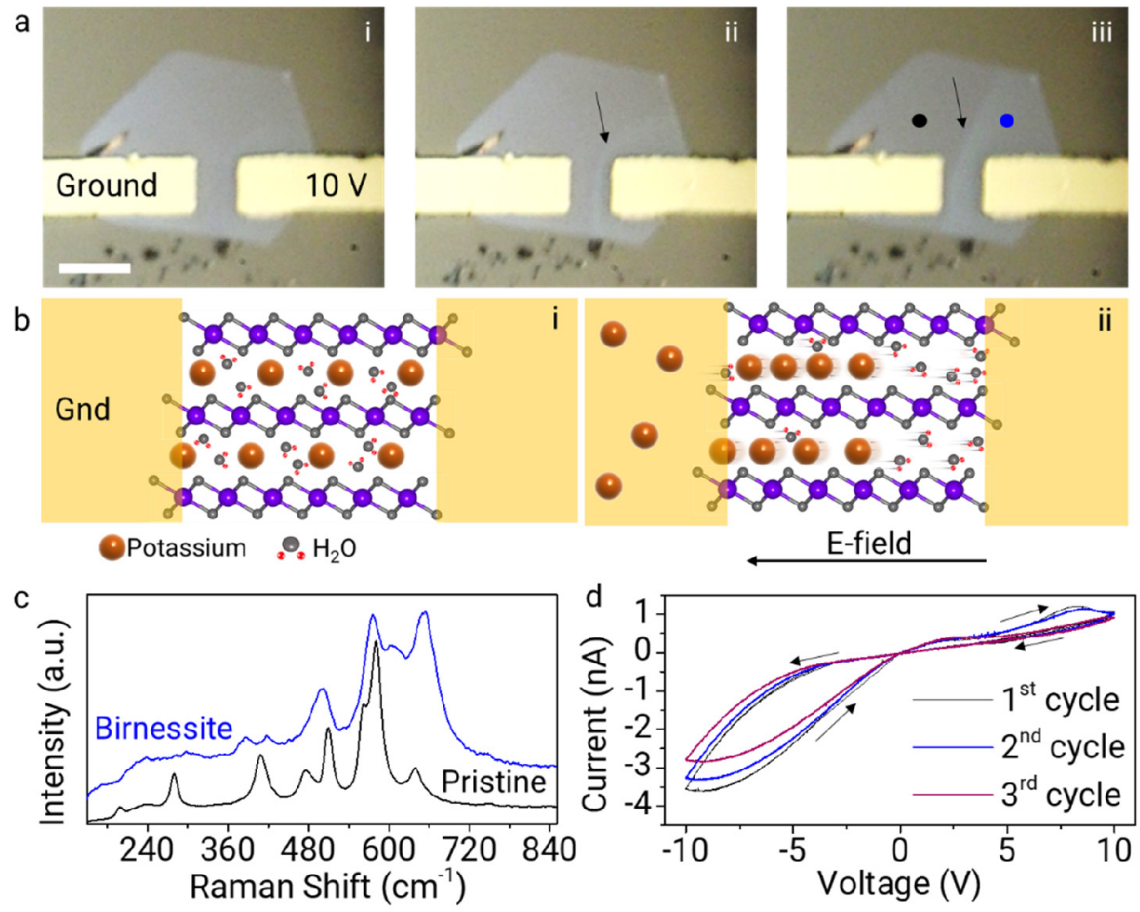

Figure 4. (a) A series of optical microscope images taken under $10 \mathrm{~V}$ bias over the course of 20 min shows the appearance of a bright contrast region near the positive electrode. Panel a-i shows the image captured when the bias is applied, panel a-ii after 10 min, and panel a-iii after 20 min. The black arrows point to the boundary of the bright and dark contrast regions. Black and blue dots marked on the image indicate the positions that Raman spectra are taken from. Crystal thickness is $6.6 \mathrm{~nm}$. Scale bar is $10 \mu \mathrm{m}$. (b) Raman spectra taken from the different contrast regions of the crystal under bias as marked in (a-iii) with black and blue dots matches with the pristine (black curve) and with the birnessite (blue curve) reported in the literature. Absence of $280 \mathrm{~cm}^{-1}$ in the spectra from the bright contrast region indicates no $\mathrm{K}-\mathrm{O}$ stretching. (c) Three consecutive $I-V$ cycles taken from a device after application of $16 \mathrm{~V}$. A broad hysteresis in a clockwise direction appears. This can be explained by the sluggish response of ion movement from the contacts to the material and separation of water and potassium within the material. The large asymmetry in the $I-V$ curve can be explained by the asymmetry of the contact configuration. (d) Schematics depict the condition when panel i with no bias is applied and panel ii when bias is applied. The potassium ions and their hydration layer become separated under the electric field. Accordingly, the bright contrast region on the right electrode corresponds to the birnessite formation with no potassium ions.

$\mathrm{Mn}-\mathrm{O}$ bonds is typically missing in the Raman spectra reported in the literature as the disorder in the hydrothermally synthesized crystals cause broadening of the Raman features. ${ }^{34}$ X-ray photoelectron spectroscopy (XPS) was used to identify the chemical composition and related valence states of $\mathrm{Mn}$ (Figure 2d). Mn 2p scan mainly consists of a spin-orbit doublet corresponding to the $M n 2 p_{3 / 2}$ and $M n 2 p_{1 / 2}$ states around the binding energy of 642.2 and $653.9 \mathrm{eV}$. To identify $\mathrm{Mn}$ oxidation states, the binding energy of $\mathrm{Mn} 2 \mathrm{p}_{3 / 2}$ is considered, which increases progressively as the oxidation state of $\mathrm{Mn}$ increases. ${ }^{43}$ The asymmetrical $\mathrm{Mn} \mathrm{2} 2 \mathrm{p}_{3 / 2}$ peak is deconvoluted into two peaks at 641.8 and $642.9 \mathrm{eV}$, corresponding to $\mathrm{Mn}^{3+}$ and $\mathrm{Mn}^{4+}$, respectively. ${ }^{44-46}$ The presence of $\mathrm{Mn}^{3+}$ oxidation state compensates the charge deficit in octahedral layers of $\mathrm{Mn}^{4+}$ along with the $\mathrm{K}$ ions. ${ }^{47}$ This point is also supported by the binding energy difference $(\Delta E)$ of $\mathrm{Mn} 3 \mathrm{~s}$ multiplet splitting as the splitting energy $\Delta E$ is used to qualitatively determine the oxidation state of Mn (ref 48.). The obtained $\Delta E$ value is $\sim 5.1 \mathrm{eV}$ supporting mixed oxidation state nature of $\mathrm{Mn}$ in $2 \mathrm{D} \mathrm{K}-\mathrm{MnO}_{2}$ (Figure S3). The $\mathrm{O} 1 \mathrm{~s}$ scan shows a single peak around $530.2 \mathrm{eV}$ which is dominantly coming from the sapphire substrate. ${ }^{49} \mathrm{~K} 2 \mathrm{p}$ survey exhibits the $\mathrm{K} 2 \mathrm{p}_{3 / 2}$ and $\mathrm{K} 2 \mathrm{p}_{1 / 2}$ peaks at 292.2 and $295.0 \mathrm{eV}$, respectively. These peaks are assigned to potassium species residing between the $\mathrm{MnO}_{6}$ octahedral-based sheets.

Now, we would like to turn our attention to the charge transport properties of $2 \mathrm{D} \mathrm{K}-\mathrm{MnO}_{2}$ in ambient. First, we started our measurements on devices with gold electrodes, patterned using standard lithography techniques (see Supporting Information for details). Current-voltage $(I-V)$ curves from gold contacted crystals show a linear $I-V$ with typical resistances ranging in giga-ohms (Figure 3a). To elucidate the dominant charge carrier type, we performed voltage-time ( $V-$ $t)$ measurements under constant current as shown in Figure 3b. Upon application of the constant current, the voltage across the contacts increases with an exponential decay. Similarly, when the current is removed, the voltage across the contacts decays exponentially. In both cases, the experimental data can be fitted perfectly with the empirical formula $V(t)=$ $\left.V_{0}+V_{1} e^{-t / \lambda}\right)$ where, $V_{0}$ and $V_{1}$ are some fitting parameters and $\lambda$ is the time constant for the exponential decay function. Both for the rise and the decay, we obtain the same $\lambda$-value within an error margin. More significantly, $\lambda$ is a function of the crosssectional contact area of the crystal, $\sigma c=w \cdot d$ with the ground electrode, where $w$ and $d$ are the width and thickness of the crystal, respectively. The ratio of the $\lambda / \sigma_{\mathrm{c}}$ is constant for the asfabricated devices. The ratio for three different devices is $81 \pm$ $10 \mathrm{~s} / \mu \mathrm{m}^{2}$. This shows that the decay and rise of the voltage across the contacts is determined by a phenomenon that the contact area with the ground electrode is important. Another observation we would like to note is that for a device with pure electronic conductivity the contact cross-sectional area should have no effect in charge transport in micrometer scales at room temperature and $\lambda$ should be less than a millisecond. For mixed 

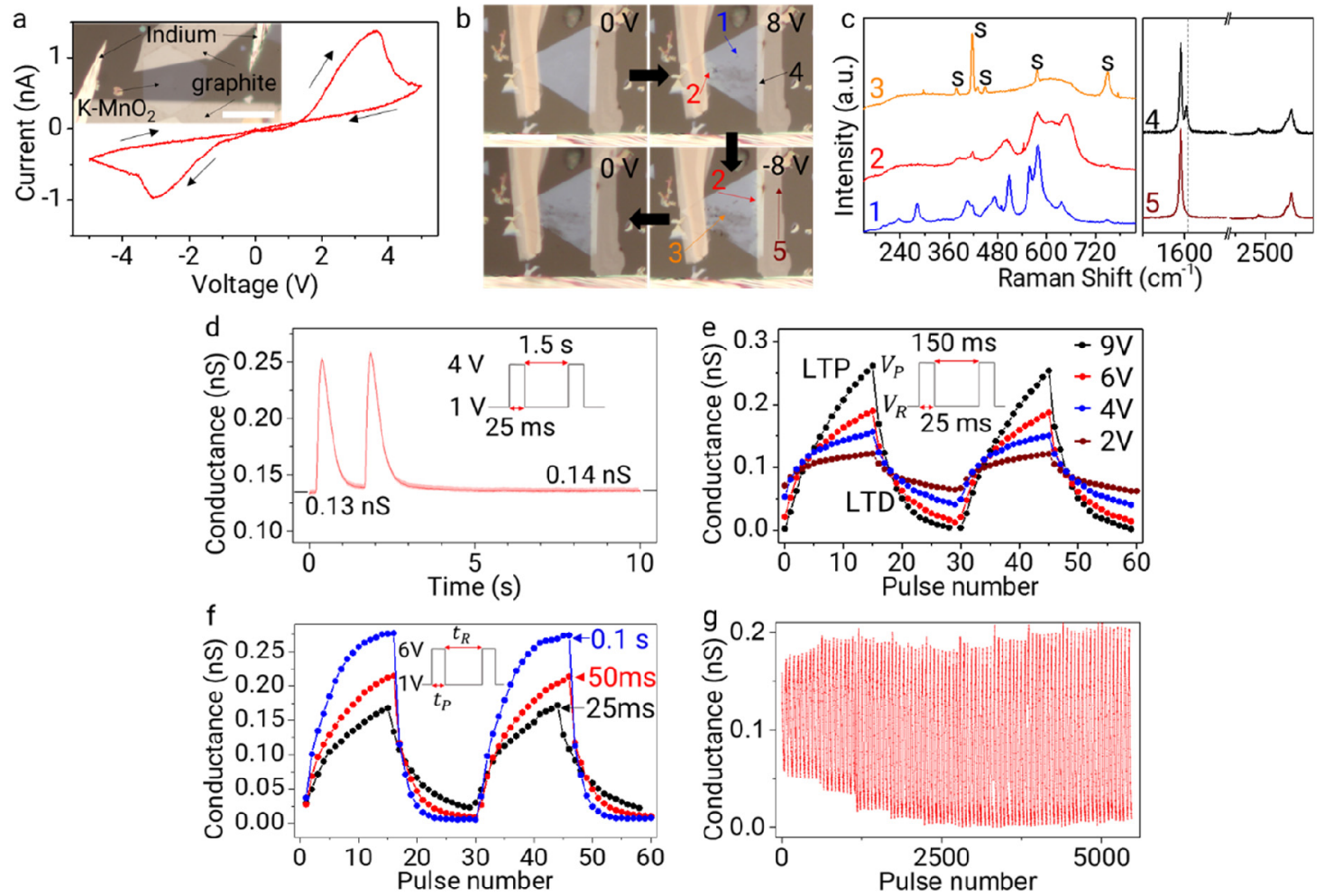

Figure 5. (a) $I-V$ cycle from a graphite/ $\mathrm{K}-\mathrm{MnO}_{2} /$ graphite memristive device. Sweep direction is indicated with black arrows on the $I-V$ curve. Inset shows a typical device. Scale bar is $20 \mu \mathrm{m}$. (b) Optical microscope images taken during the $I-V$ cycling shows optical contrast on $\mathrm{K}_{-} \mathrm{MnO}_{2}$ at different biases. Right graphite electrode is the ground. Upper left panel shows the pristine crystal. Following the black arrows, upper right panel shows the contrast change at $8 \mathrm{~V}$. Lower right panel is at $-8 \mathrm{~V}$ and lower left panel is after cycling back to $0 \mathrm{~V}$. Colored numbers with arrows show the positions where Raman spectra are collected on the sample during the $I-V$ cycling. Scale bar is $20 \mu \mathrm{m}$. (c) Raman spectra collected from points marked on (b) blue line (1) matches with the Raman peaks of the pristine $\mathrm{K}_{-} \mathrm{MnO}_{2}$. Red line (2) taken from near the positive electrode (i.e., the electrode with higher potential) shows the birnessite Raman. Absence of low-frequency vibrational bands indicate that potassium ions are missing in the structure. Orange line (3) taken from the less contrasty region near the negative electrode shows a very broad Raman band near $600 \mathrm{~cm}^{-1}$. All the sharp peaks in the spectrum belongs to the sapphire substrate, marked with "s". Black line (4) is taken from the graphite electrode over the crystal. The peak indicated with a gray dashed line shows the Raman band corresponding to $\mathrm{KC}_{72}$. Maroon line (5) shows the Raman spectrum over graphite at a distance to the crystal. $\mathrm{KC}_{72}$ peak is missing. (d) Short-term potentiation of the device with $26 \mathrm{~nm}$ crystal shows a gradual change in the conductance of the $\mathrm{K}-\mathrm{MnO}_{2}$ memristor. The inset shows the pulse train. The conductance value is read with $1 \mathrm{~V}$. Two spikes that are $1.5 \mathrm{~s}$ apart at $4 \mathrm{~V}$ with $25 \mathrm{~ms}$ temporal width are sent to induce the change. (e) Long-term potentiation (LTP) and depression (LTD) are shown in the figure. LTP is shown with pulse voltage $V_{\mathrm{P}}$ and LTD with $-V_{\mathrm{P}}$ while the read voltage $V_{\mathrm{R}}=-1 \mathrm{~V}$. Pulses are separated by $150 \mathrm{~ms}$ and last for $25 \mathrm{~ms}$. (f) Effect of pulse duration on LTP and LTD is shown. Longer pulse durations result in more significant facilitation of the synapse. (g) Conductance modulation via LTP and LTD shows endurance up to 5600 pulses.

ion-electron conductors, however, due to the relatively slow motion of ions voltage build up across the contacts is a slower process that may last several minutes.

As the contact cross-sectional area of the crystal determines the decay time constant for a crystal, we hypothesize that the contact electrodes are involved in the charge transport across $\mathrm{K}-\mathrm{MnO}_{2}$. The potassium ions can enter electrodes reversibly, and the electrodes act as a reservoir for the potassium ions. Upon application of bias, this reservoir can release or collect ions depending on the bias polarity. To prove the point, we performed energy dispersive X-ray spectroscopy (EDX) on the electrodes of a device with positive bias applied with respect to the ground. EDX maps show the presence of $\mathrm{K}$ in the ground electrode (Figure 3c). This is consistent with the fact that the electric field formed within the material drives the positively charged $\mathrm{K}$ ions from the positive terminal to the ground terminal.

When $\mathrm{K}-\mathrm{MnO}_{2}$ is biased with a moderate voltage $(\sim 10 \mathrm{~V})$, optical contrast difference across the crystal becomes visible. Figure 4a shows a series of optical microscope images taken over the course of $20 \mathrm{~min}$ when the device is biased with $10 \mathrm{~V}$ across the contacts. A bright region near the positive electrode appears after the application of bias and expands toward the ground terminal. This bright region disappears when the bias is removed and can reappear near the ground terminal if a negative bias is applied. The bright contrast region shows a distinctly different Raman spectrum than the pristine crystal as shown in Figure $4 \mathrm{~b}$ and corresponds to the Raman spectrum reported on the synthetic birnessite crystals with only water intercalated across the layers. ${ }^{42}$ This can be explained by separation of the hydration layer around the potassium ions as evident from the diminished low-wavenumber Raman band around $280 \mathrm{~cm}^{-1}$ that can be attributed to the stretching modes of $\mathrm{KO}_{6}$ groups. ${ }^{42}$ Such structural changes cause hysteresis in the $I-V$ curves. When we apply large bias (16 $\mathrm{V})$ to a gold contacted device, a significant clockwise hysteresis appears in the $I-V$ curve. Figure $4 c$ shows three consecutive $I-V$ cycles. When the bias is removed, crystal reverts to the pristine phase as depicted in Figure $4 \mathrm{~d}$. We would like to note that the ambient moisture can intercalate the crystals and has a significant effect on the charge transport and structural 
properties of $\mathrm{K}^{-\mathrm{MnO}_{2}}$ (see Supporting Information, Figure S4-8).

At this stage, we hypothesize that if we can induce severe depletion of $\mathrm{K}$ ions from the structure in the presence of ambient moisture, we can induce layered-to-spinel phase transition. ${ }^{32,50,51}$ An example of such a dramatic phase transition is experimentally demonstrated in Figure 1c on highly biased $\mathrm{K}-\mathrm{MnO}_{2}$. If an electrode material with more affinity toward potassium is chosen, the bias at which the charge depletion takes place can be lowered. For this reason, we used multilayer (ML) graphene as the electrode material. ML graphene can reversibly take alkali ions to form alkalicarbons and modulate the charge transport by causing reversible phase transitions. ${ }^{52}$ We exfoliated ML graphene electrodes from bulk graphite using the sticky tape method and transferred the graphene flakes over the $\mathrm{K}-\mathrm{MnO}_{2}$ crystals via a deterministic stamping method. Then, indium needles are placed at elevated temperatures for electrical contacts. Details of the device fabrication are given in the Supporting Information. A significant hysteresis as compared to the gold electrode devices is observed in graphene electrode $\mathrm{K}-\mathrm{MnO}_{2}$ devices.

To understand the mechanisms leading to the hysteresis, we optically tracked the changes on devices and performed in situ Raman spectroscopy during the $I-V$ cycling in ambient (in vacuo studies are given in the Supporting Information). Figure 5 a shows a typical $I-V$ of a graphene contacted $\mathrm{K}-\mathrm{MnO}_{2}$ device with one electrode fixed to the ground while the voltage is swept through the other electrode. Real time optical microscope images recorded during $I-V$ cycling show significant optical changes in the crystal (Figure 5b, SI Video 2 ). Beyond a certain voltage, the device exhibits negative differential resistance (NDR). The current through the device decreases with the increasing voltage, and the device switches from low resistance state (LRS) to high resistance state (HRS). We attribute this switching and NDR to two factors. First, potassium ions are partially depleted in the crystal as they migrate to the ML graphene electrodes and cannot contribute to the charge transport. Second, depletion of hydrated potassium from the certain regions of the crystal collapses the layered structure and results in a transition to spinel-like structure. $^{50}$ The first point is proven via Raman spectra collected from the graphene electrode after applying bias to the device (Figure 5c). The spectrum shows the formation of potassium graphite with the Raman spectrum matching with $\mathrm{KC}_{72}$ reported in the literature, confirming the intercalation of potassium. ${ }^{52} \mathrm{~A}$ darker contrast region appears over the crystal in the optical image. Raman spectrum from these regions show a broad spectrum that can be attributed to mixture of various structures (Figure S9a). When this dark region is illuminated for several minutes with high intensity laser beam, a sharp Raman peak gradually forms that can be attributed to the formation of like $\mathrm{Mn}_{3} \mathrm{O}_{4}$ (ref 30). Figure S9b shows the evolution of the Raman peaks upon illumination with a few $\mathrm{mW} 525 \mathrm{~nm}$ laser beam. To mimic a similar structure, we transferred crystals over TEM grids and illuminated them with high intensity laser beam (Figure S9c). The gradual change in the contrast of the crystal and the Raman spectrum matches with the biased crystals (Figure S9d). HR-TEM from the illuminated regions show formation of islands with different crystal structure (Figure $\mathrm{S} 9 \mathrm{e}-\mathrm{h}$ ). EDS spectrum from the illuminated regions shows no potassium (Figure S9i-k).
When the bias is lowered, no significant change is observed optically until the negative biases. Once the voltage is further decreased, the current increases in the opposite direction to a maximum followed by NDR. This indicates the reversal of the spinel-like structure near the ground electrode to the layered birnessite structure and the transition is observed optically. I$V$ measurements and Raman spectra taken from the biased crystals (Figure S7) show that for such a reverse transition moisture from the ambient is required. ${ }^{19}$ As the bias is reversed, potassium ions migrate from the ground electrode to the crystal. This creates a charge imbalance in the structure and attracts moisture in the ambient to compensate the deficit. ${ }^{31}$ Strikingly, when the devices are $I-V$ cycled many times in the ambient, small droplets appear near the electrodes (Figure $S 10)$. Moreover, for devices $I-V$ cycled at elevated temperatures or under vacuum the hysteresis diminishes (Figure S11). These observations are consistent with the fact that potassium depleted from the structure requires ambient moisture to enter the crystal again.

So far, in this Letter we showed that $2 \mathrm{D} \mathrm{K}-\mathrm{MnO}_{2}$ with multilayer graphene contacts intrinsically exhibits both the ionic transport and ionic modulation properties. Ionic transport and modulation in $2 \mathrm{D}$ materials can be useful in biomimicking neuromorphic devices. ${ }^{4}$ Synapses control the passage of electrical or chemical signals across neurons and play a central role in information processing and storage of neural networks. ${ }^{53}$ Mimicking synapses with artificial components may enable integration of large-scale neural networks with low-power consumption. To demonstrate the neuromorphic capabilities of $\mathrm{K}-\mathrm{MnO}_{2}$ based devices, we conducted a series of experiments including potentiation, depression, and endurance. First, the short-term potentiation (STP) of the devices is evaluated (Figure 5d). Two pulses of $25 \mathrm{~ms}$ temporal width at pulsing voltage $V_{\mathrm{P}}=4 \mathrm{~V}$ separated by $1.5 \mathrm{~s}$ causes a gradual change in the conductance of the device, that is, facilitation of the synapse. Read voltage $V_{\mathrm{R}}$ is set to $1 \mathrm{~V}$ to prevent changes in the resistance state of the device. Longterm potentiation (LTP) and depression (LTD) are also evident in $\mathrm{K}-\mathrm{MnO}_{2}$ devices. Conductance of the device can be altered gradually through application of voltage pulse trains. Depending on the amplitude (Figure 5e) and the duration of the pulses (Figure 5f), the devices show varying degrees of modulation of the conductance. Potentiation and depotentiation repeated over more than 5500 cycles show the endurance of the devices upon cycling (Figure $5 \mathrm{~g}$ ). Finally, we would like to comment on ionic coupling of memristors via a common graphite electrode. In principle, it is possible to use graphite as an ion transport channel across memristors to mimic synaptic competition and cooperation behaviors. We illustrated synaptic competition in a two-synapse configuration device (Figure S12). A more detailed study is required to elucidate the mechanisms underlying the coupling effect.

In summary, we introduced spontaneously ion intercalated $2 \mathrm{D} \mathrm{K}_{-} \mathrm{MnO}_{2}$ large area single crystals down to a few nanometers and demonstrated reversible electric field induced phase transitions. We characterized the fundamental properties of the $2 \mathrm{D}$ crystals and explained the mechanisms leading to the phase transitions. We utilized the observed intriguing ionic charge transport coupled with structural changes in memristive applications and demonstrated that these memristors can be used in neuromorphic applications. Our results here also may shed some light on the energy storage and releasing properties of $\mathrm{MnO}_{2}$. Moreover, our observations on the electric field 
controllable optical contrast change in $\mathrm{K}-\mathrm{MnO}_{2}$ could be used in optical switching applications. Manganese is an abundant material and a cheaper alternative to many other transition metals. A 2D large scale form of manganese oxide family could be significant in many applications including energy storage, catalysis, and water splitting. Finally, $\mathrm{MnO}_{2}$ sheets with different intercalants such as $\mathrm{Li}, \mathrm{Na}, \mathrm{Mg}$, and so forth can be synthesized in large areas using similar methods employed in this letter for catalysis and supercapacitor applications.

\section{ASSOCIATED CONTENT}

\section{SI Supporting Information}

The Supporting Information is available free of charge at https://pubs.acs.org/doi/10.1021/acs.nanolett.1c00735.

$\mathrm{K}-\mathrm{MnO}_{2}$ synthesis, device fabrication, and experimental details; additional figures S1-S12: optical microscope micrographs, Raman spectra, device stability, XPS measurements, detailed discussion on the effects of ambient moisture on $\mathrm{K}-\mathrm{MnO}_{2}$, TEM and SAED measurements, two-synapse device measurements (PDF)

Movie of applying large bias over a single crystal K$\mathrm{MnO}_{2}$ (MOV)

Movie of crystal growth in real time (MOV)

Movie of real time optical microscopy of $I-V$ cycling of a graphite contacted $\mathrm{K}-\mathrm{MnO}_{2}(\mathrm{MP} 4)$

\section{AUTHOR INFORMATION}

\section{Corresponding Authors}

Talip Serkan Kasirga - Institute of Materials Science and Nanotechnology, Bilkent University UNAM, Ankara 06800, Turkey; Department of Physics, Bilkent University, Ankara 06800, Turkey; 이이.org/0000-0003-3510-5059; Email: kasirga@unam.bilkent.edu.tr

Kibum Kang - Department of Materials Science and Engineering, Korea Advanced Institute of Science and Technology (KAIST), Daejeon 34141, Republic of Korea; ○ orcid.org/0000-0003-1674-1826; Email: kibum.kang@ kaist.ac.kr

\section{Authors}

Hamid Reza Rasouli - Institute of Materials Science and Nanotechnology, Bilkent University UNAM, Ankara 06800, Turkey

Jeongho Kim - Department of Materials Science and Engineering, Korea Advanced Institute of Science and Technology (KAIST), Daejeon 34141, Republic of Korea

Naveed Mehmood - Institute of Materials Science and Nanotechnology, Bilkent University UNAM, Ankara 06800, Turkey

Ali Sheraz - Department of Physics, Bilkent University, Ankara 06800, Turkey

Min-kyung Jo - Korea Research Institute of Standards \& Science (KRISS), Daejeon 34113, Republic of Korea; Department of Materials Science and Engineering, Korea Advanced Institute of Science and Technology (KAIST), Daejeon 34141, Republic of Korea

Seungwoo Song - Korea Research Institute of Standards \& Science (KRISS), Daejeon 34113, Republic of Korea; (1) orcid.org/0000-0002-8524-3444

Complete contact information is available at: https://pubs.acs.org/10.1021/acs.nanolett.1c00735

\section{Author Contributions}

T.S.K. conceived the experiments. H.R.R. performed the crystal synthesis, fabricated devices, and performed the measurements with T.S.K. A.S. and N.M. fabricated devices, J.H. and K.K. performed TEM, FT-IR, and some XPS measurements. M.J. and S.S. performed the vacuum XRD measurements. T.S.K. wrote the manuscript and all authors commented on the manuscript.

\section{Notes}

The authors declare no competing financial interest.

\section{ACKNOWLEDGMENTS}

T.S.K. acknowledges support from TUBITAK under Grant 116M226. H.R.R. acknowledges support from TUBITAK under Grant 120F048. K.K. acknowledges the support from the National Research Foundation of Korea (NRF2020K2A9A1A06108923) and the Korea Institute of Science and Technology (KIST) Institutional Program (2 V07080-19P148). T.S.K. is grateful to Aykut Erbaş for useful discussions and comments on the manuscript.

\section{REFERENCES}

(1) Zhang, F.; Zhang, H.; Krylyuk, S.; Milligan, C. A.; Zhu, Y.; Zemlyanov, D. Y.; Bendersky, L. A.; Burton, B. P.; Davydov, A. V.; Appenzeller, J. Electric-Field Induced Structural Transition in Vertical MoTe2- and Mo1-XWxTe2-Based Resistive Memories. Nat. Mater. 2019, 18 (1), 55-61.

(2) Liu, G.; Debnath, B.; Pope, T. R.; Salguero, T. T.; Lake, R. K.; Balandin, A. A. A Charge-Density-Wave Oscillator Based on an Integrated Tantalum Disulfide-Boron Nitride-Graphene Device Operating at Room Temperature. Nat. Nanotechnol. 2016, 11 (10), 845-850.

(3) Acerce, M.; Voiry, D.; Chhowalla, M. Metallic 1T Phase MoS2 Nanosheets as Supercapacitor Electrode Materials. Nat. Nanotechnol. 2015, 10 (4), 313-318.

(4) Zhu, X.; Li, D.; Liang, X.; Lu, W. D. Ionic Modulation and Ionic Coupling Effects in MoS2 Devices for Neuromorphic Computing. Nat. Mater. 2019, 18 (2), 141-148.

(5) Wan, J.; Lacey, S. D.; Dai, J.; Bao, W.; Fuhrer, M. S.; Hu, L. Tuning Two-Dimensional Nanomaterials by Intercalation: Materials, Properties and Applications. Chem. Soc. Rev. 2016, 45 (24), 67426765.

(6) Zhang, J.; Yang, A.; Wu, X.; van de Groep, J.; Tang, P.; Li, S.; Liu, B.; Shi, F.; Wan, J.; Li, Q.; Sun, Y.; Lu, Z.; Zheng, X.; Zhou, G.; Wu, C.-L.; Zhang, S.-C.; Brongersma, M. L.; Li, J.; Cui, Y. Reversible and Selective Ion Intercalation through the Top Surface of Few-Layer MoS2. Nat. Commun. 2018, 9 (1), 5289.

(7) Park, J.; Kim, J.-S.; Park, J.-W.; Nam, T.-H.; Kim, K.-W.; Ahn, J.H.; Wang, G.; Ahn, H.-J. Discharge Mechanism of MoS2 for Sodium Ion Battery: Electrochemical Measurements and Characterization. Electrochim. Acta 2013, 92, 427-432.

(8) Wan, J.; Bao, W.; Liu, Y.; Dai, J.; Shen, F.; Zhou, L.; Cai, X.; Urban, D.; Li, Y.; Jungjohann, K.; Fuhrer, M. S.; Hu, L. In Situ Investigations of Li-MoS2 with Planar Batteries. Adv. Energy Mater. 2015, 5 (5), 1401742.

(9) Xiong, F.; Wang, H.; Liu, X.; Sun, J.; Brongersma, M.; Pop, E.; Cui, Y. Li Intercalation in MoS 2: In Situ Observation of Its Dynamics and Tuning Optical and Electrical Properties. Nano Lett. 2015, 15 (10), 6777-6784.

(10) Yu, Y.; Yang, F.; Lu, X. F.; Yan, Y. J.; Cho, Y.-H.; Ma, L.; Niu, X.; Kim, S.; Son, Y.-W.; Feng, D.; Li, S.; Cheong, S.-W.; Chen, X. H.; Zhang, Y. Gate-Tunable Phase Transitions in Thin Flakes of 1TTaS2. Nat. Nanotechnol. 2015, 10 (3), 270-276.

(11) Yao, J.; Koski, K. J.; Luo, W.; Cha, J. J.; Hu, L.; Kong, D.; Narasimhan, V. K.; Huo, K.; Cui, Y. Optical Transmission 
Enhacement through Chemically Tuned Two-Dimensional Bismuth Chalcogenide Nanoplates. Nat. Commun. 2014, 5 (1), 5670.

(12) Wang, Y.; Ou, J. Z.; Balendhran, S.; Chrimes, A. F.; Mortazavi, M.; Yao, D. D.; Field, M. R.; Latham, K.; Bansal, V.; Friend, J. R.; Zhuiykov, S.; Medhekar, N. V.; Strano, M. S.; Kalantar-zadeh, K. Electrochemical Control of Photoluminescence in Two-Dimensional MoS 2 Nanoflakes. ACS Nano 2013, 7 (11), 10083-10093.

(13) Ou, J. Z.; Chrimes, A. F.; Wang, Y.; Tang, S. Y.; Strano, M. S.; Kalantar-Zadeh, K. Ion-Driven Photoluminescence Modulation of Quasi-Two-Dimensional MoS 2 Nanoflakes for Applications in Biological Systems. Nano Lett. 2014, 14 (2), 857-863.

(14) Gong, Y.; Yuan, H.; Wu, C.-L.; Tang, P.; Yang, S.-Z.; Yang, A.; Li, G.; Liu, B.; van de Groep, J.; Brongersma, M. L.; Chisholm, M. F.; Zhang, S.-C.; Zhou, W.; Cui, Y. Spatially Controlled Doping of TwoDimensional SnS2 through Intercalation for Electronics. Nat. Nanotechnol. 2018, 13 (4), 294-299.

(15) Cha, J. J.; Koski, K. J.; Huang, K. C. Y.; Wang, K. X.; Luo, W.; Kong, D.; Yu, Z.; Fan, S.; Brongersma, M. L.; Cui, Y. TwoDimensional Chalcogenide Nanoplates as Tunable Metamaterials via Chemical Intercalation. Nano Lett. 2013, 13 (12), 5913-5918.

(16) Li, K.; Chang, T. H.; Xie, Q.; Cheng, Y.; Yang, H.; Chen, J.; Chen, P. Y. Tunable Magnetic Response in 2D Materials via Reversible Intercalation of Paramagnetic Ions. Adv. Electron. Mater. 2019, 5 (6), 1900040.

(17) Wang, J.; Zhang, G.; Zhang, P. Layered Birnessite-Type MnO2 with Surface Pits for Enhanced Catalytic Formaldehyde Oxidation Activity. J. Mater. Chem. A 2017, 5 (12), 5719-5725.

(18) Ghasemian, M. B.; Mayyas, M.; Idrus-Saidi, S. A.; Jamal, M. A.; Yang, J.; Mofarah, S. S.; Adabifiroozjaei, E.; Tang, J.; Syed, N.; O'Mullane, A. P.; Daeneke, T.; Kalantar-Zadeh, K. Self-Limiting Galvanic Growth of $\mathrm{MnO} 2$ Monolayers on a Liquid Metal-Applied to Photocatalysis. Adv. Funct. Mater. 2019, 29 (36), 1901649.

(19) Nam, K. W.; Kim, S.; Lee, S.; Salama, M.; Shterenberg, I.; Gofer, Y.; Kim, J. S.; Yang, E.; Park, C. S.; Kim, J. S.; Lee, S. S.; Chang, W. S.; Doo, S. G.; Jo, Y. N.; Jung, Y.; Aurbach, D.; Choi, J. W. The High Performance of Crystal Water Containing Manganese Birnessite Cathodes for Magnesium Batteries. Nano Lett. 2015, 15 (6), 4071-4079.

(20) Shan, X.; Guo, F.; Charles, D. S.; Lebens-Higgins, Z.; Abdel Razek, S.; Wu, J.; Xu, W.; Yang, W.; Page, K. L.; Neuefeind, J. C.; Feygenson, M.; Piper, L. F. J.; Teng, X. Structural Water and Disordered Structure Promote Aqueous Sodium-Ion Energy Storage in Sodium-Birnessite. Nat. Commun. 2019, 10 (1), 1-11.

(21) Post, J. E. Manganese Oxide Minerals: Crystal Structures and Economic and Environmental Significance. Proc. Natl. Acad. Sci. U. S. A. 1999, 96 (7), 3447-3454.

(22) Zhu, H. T.; Luo, J.; Yang, H. X.; Liang, J. K.; Rao, G. H.; Li, J. B.; Du, Z. M. Birnessite-Type MnO2 Nanowalls and Their Magnetic Properties. J. Phys. Chem. C 2008, 112 (44), 17089-17094.

(23) Jakubek, T.; Hudy, C.; Gryboś, J.; Manyar, H.; Kotarba, A. Thermal Transformation of Birnessite (OL) Towards Highly Active Cryptomelane (OMS-2) Catalyst for Soot Oxidation. Catal. Lett. 2019, 149 (8), 2218-2225.

(24) Shin, J.; Seo, J. K.; Yaylian, R.; Huang, A.; Meng, Y. S. A Review on Mechanistic Understanding of $\mathrm{MnO} 2$ in Aqueous Electrolyte for Electrical Energy Storage Systems. Int. Mater. Rev. 2020, 65 (6), 356387.

(25) Duan, X.; Yang, J.; Gao, H.; Ma, J.; Jiao, L.; Zheng, W. Controllable Hydrothermal Synthesis of Manganese Dioxide Nanostructures: Shape Evolution, Growth Mechanism and Electrochemical Properties. CrystEngComm 2012, 14 (12), 4196.

(26) Chen, B.-R.; Sun, W.; Kitchaev, D. A.; Mangum, J. S.; Thampy, V.; Garten, L. M.; Ginley, D. S.; Gorman, B. P.; Stone, K. H.; Ceder, G.; Toney, M. F.; Schelhas, L. T. Understanding Crystallization Pathways Leading to Manganese Oxide Polymorph Formation. Nat. Commun. 2018, 9 (1), 2553.

(27) Kitchaev, D. A.; Dacek, S. T.; Sun, W.; Ceder, G. Thermodynamics of Phase Selection in MnO 2 Framework Structures through Alkali Intercalation and Hydration. J. Am. Chem. Soc. 2017, 139 (7), 2672-2681.

(28) Yuan, Y.; He, K.; Byles, B. W.; Liu, C.; Amine, K.; Lu, J.; Pomerantseva, E.; Shahbazian-Yassar, R. Deciphering the Atomic Patterns Leading to MnO2 Polymorphism. Chem. 2019, 5 (7), 17931805.

(29) Hao, J.; Mou, J.; Zhang, J.; Dong, L.; Liu, W.; Xu, C.; Kang, F. Electrochemically Induced Spinel-Layered Phase Transition of Mn3O4 in High Performance Neutral Aqueous Rechargeable Zinc Battery. Electrochim. Acta 2018, 259, 170-178.

(30) Birkner, N.; Navrotsky, A. Rapidly Reversible Redox Transformation in Nanophase Manganese Oxides at Room Temperature Triggered by Changes in Hydration. Proc. Natl. Acad. Sci. U. S. A. 2014, 111 (17), 6209-6214.

(31) Kim, S.; Lee, S.; Nam, K. W.; Shin, J.; Lim, S. Y.; Cho, W.; Suzuki, K.; Oshima, Y.; Hirayama, M.; Kanno, R.; Choi, J. W. On the Mechanism of Crystal Water Insertion during Anomalous Spinel-toBirnessite Phase Transition. Chem. Mater. 2016, 28 (15), 5488-5494.

(32) Kim, S.; Nam, K. W.; Lee, S.; Cho, W.; Kim, J. S.; Kim, B. G.; Oshima, Y.; Kim, J. S.; Doo, S. G.; Chang, H.; Aurbach, D.; Choi, J. W. Direct Observation of an Anomalous Spinel-to-Layered Phase Transition Mediated by Crystal Water Intercalation. Angew. Chem., Int. Ed. 2015, 54 (50), 15094-15099.

(33) Omomo, Y.; Sasaki, T.; Wang, L.; Watanabe, M. Redoxable Nanosheet Crystallites of $\mathrm{MnO} 2$ Derived via Delamination of a Layered Manganese Oxide. J. Am. Chem. Soc. 2003, 125 (12), 35683575.

(34) Tu, M.; Lu, H.; Luo, S.; Peng, H.; Li, S.; Ke, Y.; Yuan, S.; Huang, W.; Jie, W.; Hao, J. Reversible Transformation between Bipolar Memory Switching and Bidirectional Threshold Switching in 2D Layered K-Birnessite Nanosheets. ACS Appl. Mater. Interfaces 2020, 12 (21), 24133-24140.

(35) Liu, Z.; Ma, R.; Ebina, Y.; Takada, K.; Sasaki, T. Synthesis and Delamination of Layered Manganese Oxide Nanobelts. Chem. Mater. 2007, 19 (26), 6504-6512.

(36) Rasouli, H. R.; Mehmood, N.; Çakıroğlu, O.; Kasırga, T. S. Real Time Optical Observation and Control of Atomically Thin Transition Metal Dichalcogenide Synthesis. Nanoscale 2019, 11 (15), $7317-7323$.

(37) Xiao, W.; Wang, D.; Lou, X. W. Shape-Controlled Synthesis of $\mathrm{MnO} 2$ Nanostructures with Enhanced Electrocatalytic Activity for Oxygen Reduction. J. Phys. Chem. C 2010, 114 (3), 1694-1700.

(38) Drits, V. A.; Silvester, E.; Gorshkov, A. I.; Manceau, A. Structure of Synthetic Monoclinic Na-Rich Birnessite and Hexagonal Birnessite; I, Results from X-Ray Diffraction and Selected-Area Electron Diffraction. Am. Mineral. 1997, 82 (9-10), 946-961.

(39) Lin, B.; Zhu, X.; Fang, L.; Liu, X.; Li, S.; Zhai, T.; Xue, L.; Guo, Q.; Xu, J.; Xia, H. Birnessite Nanosheet Arrays with High K Content as a High-Capacity and Ultrastable Cathode for K-Ion Batteries. Adv. Mater. 2019, 1900060.

(40) Chen, X.; Yan, S.; Wang, N.; Peng, S.; Wang, C.; Hong, Q.; Zhang, X.; Dai, S. Facile Synthesis and Characterization of Ultrathin $\delta$-MnO 2 Nanoflakes. RSC Adv. 2017, 7 (88), 55734-55740.

(41) Silvester, E.; Manceau, A.; Drits, V. A. Structure of Synthetic Monoclinic Na-Rich Birnessite and Hexagonal Birnessite: II. Results from Chemical Studies and EXAFS Spectroscopy. Am. Mineral. 1997, 82 (9-10), 962-978.

(42) Julien, C. Raman Spectra of Birnessite Manganese Dioxides. Solid State Ionics 2003, 159 (3-4), 345-356.

(43) Di Castro, V.; Polzonetti, G. XPS Study of MnO Oxidation. J. Electron Spectrosc. Relat. Phenom. 1989, 48 (1), 117-123.

(44) Nakayama, M.; Konishi, S.; Tagashira, H.; Ogura, K. Electrochemical Synthesis of Layered Manganese Oxides Intercalated with Tetraalkylammonium Ions. Langmuir 2005, 21 (1), 354-359.

(45) Gao, P.; Metz, P.; Hey, T.; Gong, Y.; Liu, D.; Edwards, D. D.; Howe, J. Y.; Huang, R.; Misture, S. T. The Critical Role of Point Defects in Improving the Specific Capacitance of $\delta$-MnO2 Nanosheets. Nat. Commun. 2017, 8 (1), 14559. 
(46) Dang, S.; Wen, Y.; Qin, T.; Hao, J.; Li, H.; Huang, J.; Yan, D.; Cao, G.; Peng, S. Nanostructured Manganese Dioxide with Adjustable Mn3+/Mn4+ Ratio for Flexible High-Energy Quasi-Solid Supercapacitors. Chem. Eng. J. 2020, 396, 125342.

(47) Gaillot, A.-C.; Flot, D.; Drits, V. A.; Manceau, A.; Burghammer, M.; Lanson, B. Structure of Synthetic K-Rich Birnessite Obtained by High-Temperature Decomposition of KMnO4. I. Two-Layer Polytype from $800{ }^{\circ} \mathrm{C}$ Experiment. Chem. Mater. 2003, 15 (24), $4666-4678$

(48) Ilton, E. S.; Post, J. E.; Heaney, P. J.; Ling, F. T.; Kerisit, S. N. XPS Determination of Mn Oxidation States in Mn (Hydr)Oxides. Appl. Surf. Sci. 2016, 366, 475-485.

(49) Rasouli, H. R.; Mehmood, N.; Çakıroğlu, O.; Sürmeli, E. C.; Kasirga, T. S. Synthesis of V2O3 Nanoplates for the Exploration of the Correlated Supercritical State. Phys. Rev. B: Condens. Matter Mater. Phys. 2019, 100 (16), 161107.

(50) Jo, M. R.; Kim, Y.; Yang, J.; Jeong, M.; Song, K.; Kim, Y.-I.; Lim, J.-M.; Cho, M.; Shim, J.-H.; Kim, Y.-M.; Yoon, W.-S.; Kang, Y.M. Triggered Reversible Phase Transformation between Layered and Spinel Structure in Manganese-Based Layered Compounds. Nat. Commun. 2019, 10 (1), 1-9.

(51) Yang, E.; Kim, H.; Kim, S.; Kim, I.; Kim, J.; Ji, H.; Choi, J. W.; Jung, Y. Origin of Unusual Spinel-to-Layered Phase Transformation by Crystal Water. Chem. Sci. 2018, 9 (2), 433-438.

(52) Chacón-Torres, J. C.; Wirtz, L.; Pichler, T. Manifestation of Charged and Strained Graphene Layers in the Raman Response of Graphite Intercalation Compounds. ACS Nano 2013, 7 (10), 92499259.

(53) Royer, S.; Paré, D. Conservation of Total Synaptic Weight through Balanced Synaptic Depression and Potentiation. Nature 2003, 422 (6931), 518-522. 